\title{
ATLAS RPC time-of-flight performance
}

\author{
G. Chiodini* \\ INFN Lecce (Italy) \\ E-mail: gabriele.chiodini@le.infn.it
}

On behalf of the ATLAS Collaboration.

The ATLAS Resistive Plate Chambers (RPC's) are planar large size gaseous detectors working in saturated avalanche regime with resistive electrodes and two orthogonal pick-up readout strip panels located outside a $2 \mathrm{~mm}$-thick active gas volume. In the ATLAS experiment three layers of RPC detectors are used in the ATLAS Muon Spectrometer barrel $(|\eta|<1.05)$ to generate a hardware muon trigger signal.

We show that the RPC time resolution for proton-proton collisions at $\sqrt{s}=7 \mathrm{TeV}$ with data recorded by the ATLAS detector in 2011 at LHC is about the expected one taking into account the intrinsic time resolution of a 2-mm thick RPC in avalanche mode, digitization error, electronic noise, and time-walk.

The off-line time calibration procedure and reconstruction algorithm needed to achieve the ultimate timing resolution are explained in detail. Very simple algorithms were used for off-line time calibration allowing for a possible on-line implementation in future up-grade scenarios.

The achieved time resolution can be used to suppress background hits at high luminosity and measure velocity to search for exotic slow particle from Physics beyond the Standard Model.

XI workshop on Resistive Plate Chambers and Related Detectors - RCP2012,

February 5-10, 2012

INFN Laboratori Nazionali di Frascati Italy

*Speaker. 


\section{ATLAS RPC trigger chambers}

In the ATLAS experiment [1] there are 1116 RPC single units for 26 different topologies, a total active surface of about $4000 \mathrm{~m}^{2}$, and about 330,000 electronic channels.

The muon candidates are identified by fast geometrical coincidence pattern (trigger roads) in the two measurement views $(\eta-\phi)$. This allows to provide a Region of Interest $\Delta \eta \times \Delta \phi=0.1 \times 0.1$, the highest among one of the six programmable thresholds, and the coarse measurement of the bending $(\eta)$ and non-bending $(\phi)$ coordinates, useful to seed the next on-line trigger level, in addition to the bunch crossing identification number. The on-detector trigger-readout electronics, based on the Coincidence Matrix ASIC (CMA) [2], operates with a clock frequency of $320 \mathrm{MHz}$, corresponding to eight times the frequency of the bunch crossing clock, allowing a digital time resolution of about $\frac{3.125 n s}{\sqrt{12}}=0.9 \mathrm{~ns}$. The on-line time alignment is done with tracks both for trigger hits and readout hits in order to maximize trigger efficiency inside a 25 ns trigger window.

\section{RPC time in data and simulation}

RPC has excellent time resolution due to the planar geometry, as opposite to the traditional wire chamber cylindrical geometry. In fact, in any position inside the gas volume, the constant electric field can start a charge avalanche multiplication just after the elementary ionization process takes place. The time fluctuations of the avalanche signal scale proportionally with the gas volume thickness and for ATLAS RPC this corresponds to a time resolution of about $1.2 \mathrm{~ns}$ [3].

In order to achieve in off-line data analysis the best possible time resolution it is important to establish a time calibration procedure based on algorithms that take correctly into account several effects. There are fixed delays ( due to cables, optical links, configurations ...), that need to be measured once and thereafter absorbed in a calibration constant, and track dependent delays, such as: the particle time of flight, the spread of the interaction point, the signal delay along the readout strips, which must be corrected knowing the kinematics and the geometry of the track. The signal delay along the readout strips is a relevant effect, and it is evaluated by the product of the signal propagation speed, assumed to be $208 \mathrm{~ns} / \mathrm{mm}$, and the distance from the readout electronics, given by the associated orthogonal coordinate.

In the simulation, the time of the RPC hits is emulated in such a way to reproduce the expected measurement in a perfectly timed system. The components contributing to the spread of the bare time measurements are: the time of flight of the particle from the interaction point, evaluated by GEANT4 in the particle propagation process, and the time of the signal propagation along the strip. In the simulation a Gaussian smearing of $1.5 \mathrm{~ns}$ is applied to reproduce time jitter effects measured in RPC H8 test beam [2]. A nominal time of flight, as given by a relativistic track hitting the center of the strip, is then subtracted in order to mimic the on-line time alignment procedure which inside a trigger tower maximizes the trigger efficiency compensating all relative delays.

A simple RPC standalone tracking is implemented off-line [4] and based on RPC space points, which are defined by adjacent hits (clusters) on both orthogonal views but same gas volume. The cluster time is defined as the minimum time of the adjacent hits belonging to the cluster. The tracks are straight lines defined by six space points with 1.5 average cluster size per view with $1 \mathrm{~cm}$ space resolution. A cut on the global chi2 per degree of freedom is equivalent to select high transverse 

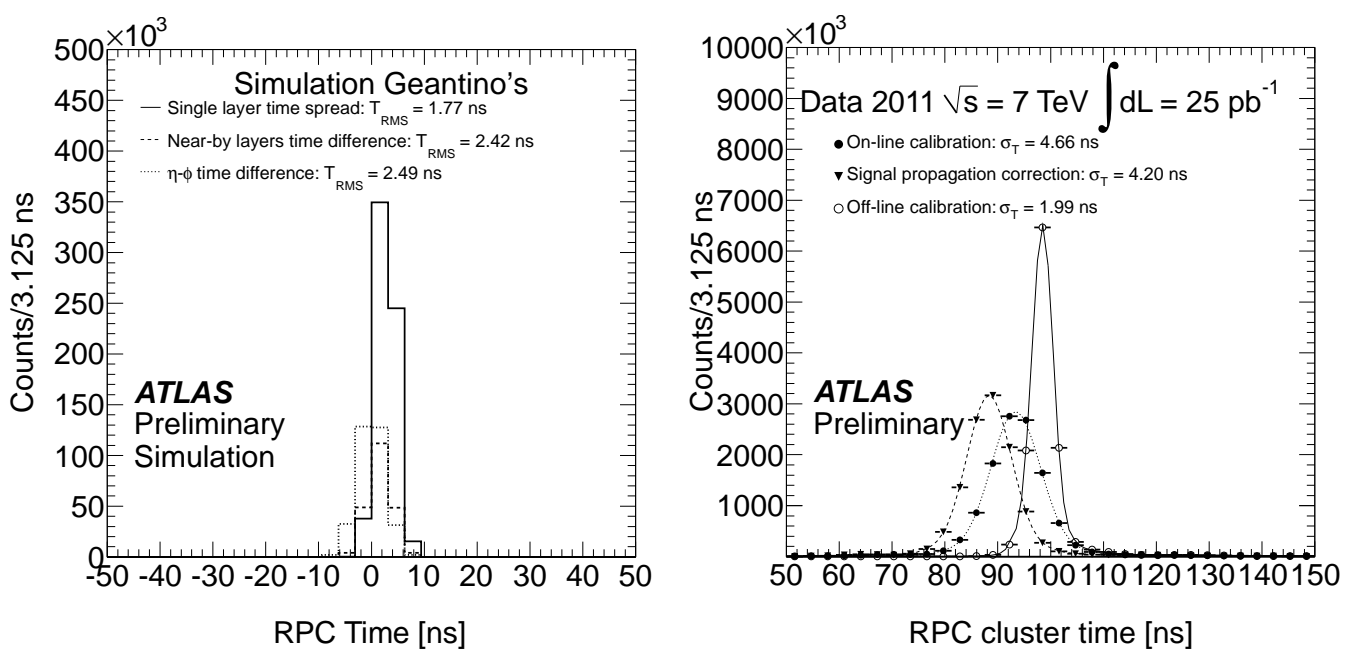

Figure 1: Left: distribution of RPC cluster time with signal time propagation along strip subtracted (continuous line), distribution of RPC cluster time difference between two near-by gas volume for the same view (dotted line), distribution of RPC cluster time difference with signal time propagation along strip subtracted between orthogonal view belonging to the same gas volume (dashed line). The RPC hits are simulated with non-interacting neutral tracks (Geantino's). Right: distribution of RPC cluster time after on-line calibration (full circles), distribution of RPC cluster time with signal delay propagation along strip subtracted (triangles), Distribution of RPC cluster time with signal delay propagation along strip subtracted after off-line calibration (open circles).

momentum tracks. To have a clean sample of muons we require that the hits contributing to the RPC track match, within 0.1 for both $\eta$ and $\phi$, the extrapolation in the RPC plane of an off-line high quality muon.

We verify the RPC time simulation and reconstruction algorithms simulating Geantino tracks without interaction point spread. Geantino's are non-interacting and neutral tracks, which leave hits in active volume but don't create secondary interactions and don't bend in magnetic fields. In Figure 1 several key distributions of RPC cluster time due to one million simulated Geantino tracks are shown. The root mean square values are reported and correspond to the values expected from a back-on-the-envelope calculation assuming a time resolution of $1.75 \mathrm{~ns}$ (1.5 ns RPC time resolution added in quadrature to the $0.9 \mathrm{~ns}$ digital time resolution) and no correlations in the time fluctuations between different gas volumes and views $(1.75 \sqrt{2}=2.47$ ns relative time spread).

\section{RPC off-line time calibration}

We assumed as off-line time calibration criteria that the arrival time of a relativistic track leaving the interaction point is in average equal to $100 \mathrm{~ns}$, this is in the center of the CMA readout window. A simple calibration algorithm is employed strip by strip. The calibration constant per strip is defined as the time shift needed to move the most probable value of the signal delay subtracted time distribution to the conventional 100 ns readout window center. In order to make sure to fulfill the calibration criteria only RPC hits matched with muon tracks are considered in the time distribution. Once the calibration constants are extracted from the data the off-line calibrated time 
must be defined consistently as the strip time, minus the signal delay, minus the calibration constant and plus the nominal $100 \mathrm{~ns}$ (this time offset is not added in the simulation). Analogously, the real time of flight is the calibrated time minus $100 \mathrm{~ns}$ and plus the nominal time of flight given by the spatial hit position with respect to the interaction points. It is worth to notice that the signal delay must always be subtracted because is just a systematic time shift that adds to the real arrival time. The 330,000 thousand calibration constants were measured adding together several runs of June 2011. The so obtained single strip off-line time resolution was stable for all channels and for all 2011 (corresponding to an integrated luminosity of about $4.8 \mathrm{fb}^{-1}$ ). Figure 1 on the right shows the achieved time resolution by the off-line calibration. The time distributions are obtained from a muon stream run not used in the calibration constant extraction. The data selection is based on RPC clusters matched with at least one extrapolated muon combined track in both views. It turn out that the time resolution obtained by on-line time alignment is of $4.7 \mathrm{~ns}$, which corresponds to a resolution of $4.2 \mathrm{~ns}$ after signal time delay subtraction, and the time resolution obtained after off-line calibration is of $2 \mathrm{~ns}$ to be compared with the ideally expected $1.75 \mathrm{~ns}$. The electronic noise and the time-walk introduced by analog and digital part coupling are expected to make-up the rest of the time resolution.

This is a very significant results because obtained for the whole ATLAS, many months of data taking, using a small calibration sample also excluded from the plots. The RPC time resolution achieved by simple off-line calibration algorithms is very near to the single unit resolution and proves that RPC detector can easily operate in standalone mode thanks to its tracking and timing capability.

\section{Background suppression and velocity measurements}

The achieved offline time resolution is very effective in reducing correlated and un-correlated background such as: loopers, beam-gas, parasitic beam-beam, beam collimator interactions , cosmic rays, and cavern background (mainly neutrons and gammas). In Figure 2 on the left the time spread between views ( $2.5 \mathrm{~ns})$ and gas volumes $(2.6 \mathrm{~ns})$ is plotted in $\log$ scale together with the arrival time. It is possible to notice a long non Gaussian tail in the arrival time also for hits matched with prompt tracks which can be strongly reduced using RPC time information's.

The velocity of particles was measured doing a linear fit between the incremental distance between spatially averaged space points and the average of the corresponding time-of-flight for each one of the 6 layers. In Figure 2 on the right the distribution of velocity measurements is shown after off-line calibration. The redundancy in the RPC trigger system and its good tracking capability allow particle velocity measurement with a precision of about $4 \%$ with IP contrain and of about $17 \%$ without it.

\section{Conclusions}

The ATLAS RPC detector reached a time resolution of about 2 ns for the whole experiment and during all 2011 data taking where the number of interactions per crossing went from about 5 to about 18 making in-time and out-time pile-up challenging. 

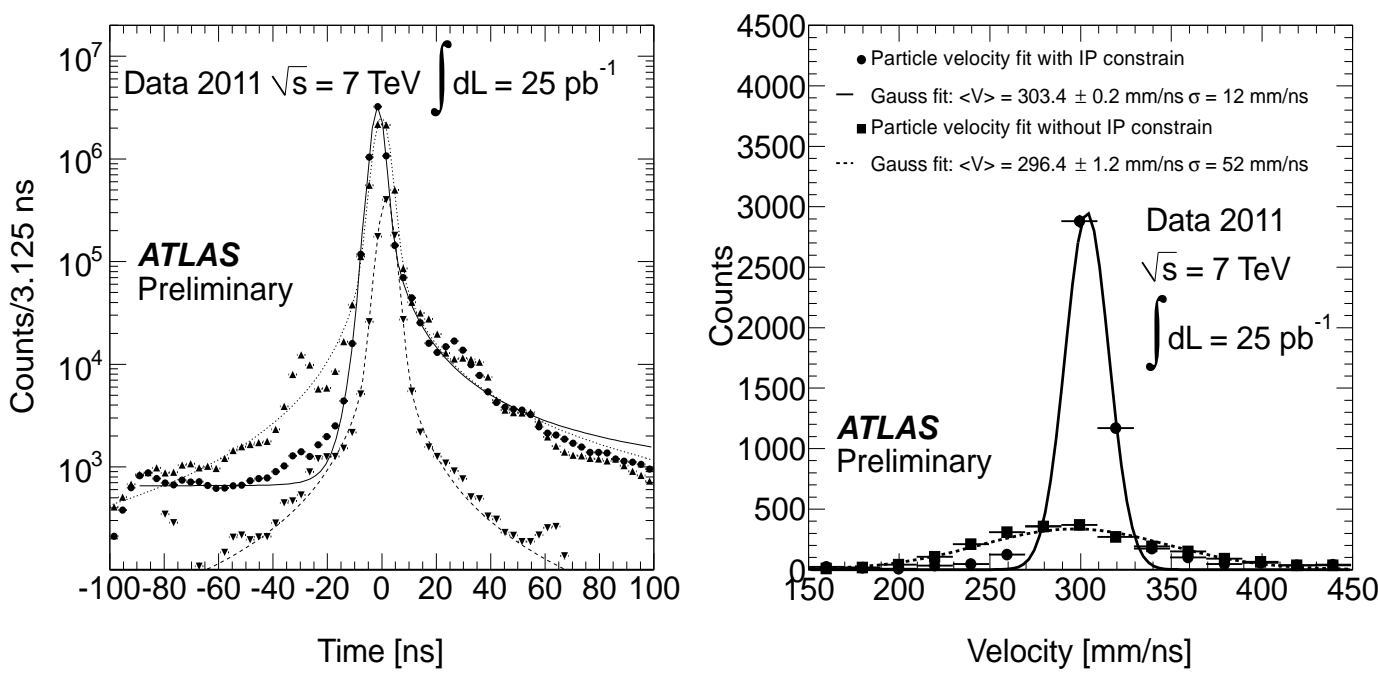

Figure 2: Left: distribution of RPC cluster time with 100 ns subtracted (circles), distribution of RPC cluster time difference between two near-by gas volume for the same view (down triangle), distribution of RPC cluster time difference between orthogonal view belonging to the same gas volume with signal time propagation along strip subtracted (up triangles). Right: distribution of velocity measured with RPC pointing track with interaction point constrain (circles) and without it (squares).

The RPC time information, having a precision an order of magnitude less than LHC bunch-crossing period, is very effective in rejecting combinatorial and cavern background already for the standalone detector, but even more powerful if combined with other detectors or full reconstruction algorithms.

\section{References}

[1] ATLAS Collaboration, "The ATLAS Experiment at the CERN Large Hadron Collider". JINST 3 S08003 (2008).

[2] ATLAS Muon Collaboration, "ATLAS Muon Spectrometer Technical Design Report". CERN/LHCC/97-22 (1997).

[3] R. Santonico R. Cardarelli, "Development of resistive plate counters". Nucl. Instrum. and Meth. 187 (1981) 377.

[4] G. Chiodini et al., "RPC cosmic ray tests in the ATLAS experiment", Nucl. Instrum. and Meth. A581 (2007) 213. 